\title{
Microprocessors in Laboratory Automation
}

\author{
M. Bos \\ Technical University Twente, Department of Chemical Technology, Enschede, The Netherlands
}

A description is given of the construction and operation of microcomputer systems, the types and functions of their various components, their programming and the peripherals and interfaces that are required to use microcomputers in a laboratory environment. The particulars of the use of microcomputers in laboratory automation are presented in some detail and illustrated by an example concerning computerization of the trace analysis of some metals.

Many of us still visualize a computer as bulky equipment occupying the better part of large rooms where the atmosphere is kept at constant temperature and humidity (Fig. 1). Nowadays, however, computers can be as small as desktop calculators, thanks to developments in the field of microelectronics (Fig. 2). The large installations still serve their purpose in number-crunching, data-base management, etc., but the superminiaturization with the technique called Large Scale Integration (LSI) that produces thousands of transistors and other electronic circuit elements on a few square millimeters of a silicon chip, can bring computing power to virtually every spot where it is needed. Accompanying the miniaturization there has been a spectacular reduction in the cost of computing power during the last decade. Together these factors are responsible for new concepts in in-

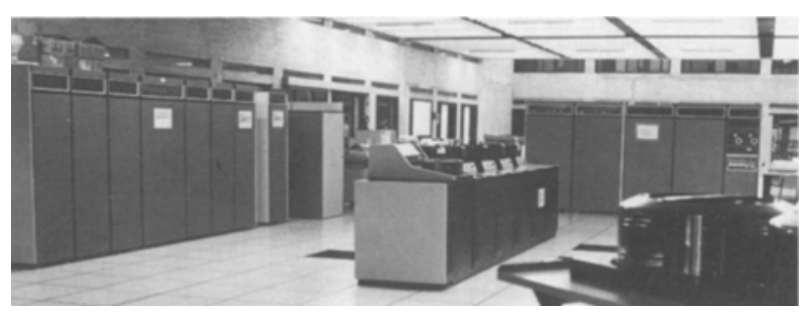

Fig. 1. Technical University Twente, Central Computerfacility strumentation and automation. A great number of measuring instruments nowadays is "smart", that is they not only provide the data of interest but also analyze these data and act on the results of these analyses.

The universal building block in these intelligent instruments is the so-called microprocessor, a complicated electronic circuit most often contained in one package having the size of about $1.5 \times 5 \mathrm{~cm}$ and from 24 to 40 connecting pins (Fig. 3). This device can perform a multitude of complex tasks for which the commands are stored in additional memory devices. The multifunctionality and great flexibility offered by this set-up enable a high degree of sophistication together with relatively short development times. For small series production of scientific instruments stan-

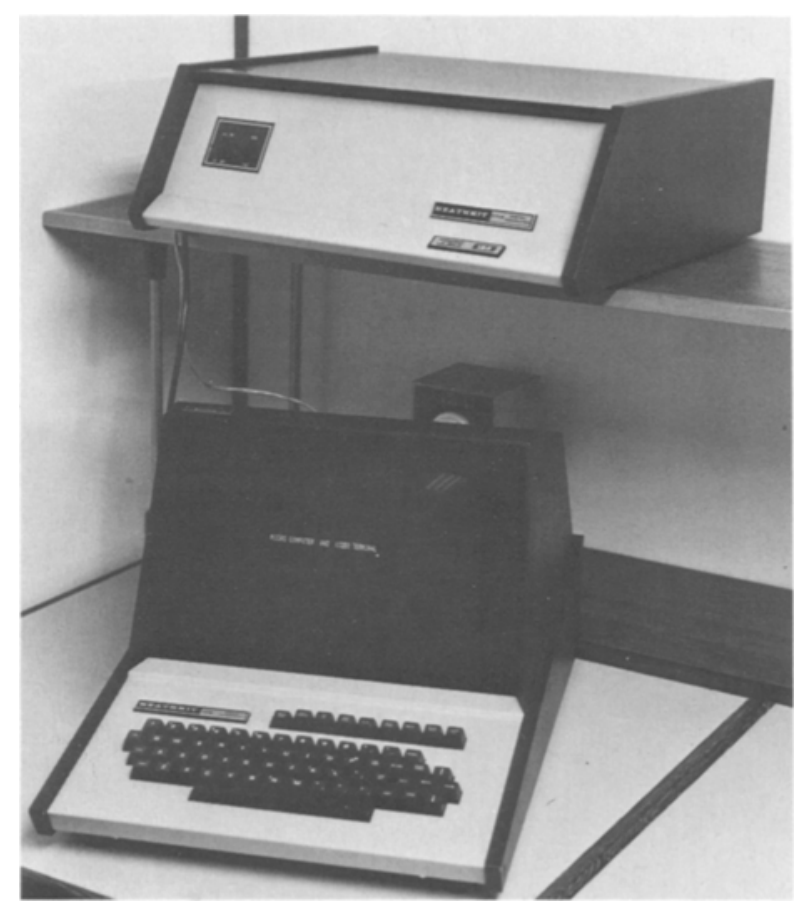

Fig. 2. Microcomputer with video terminal 


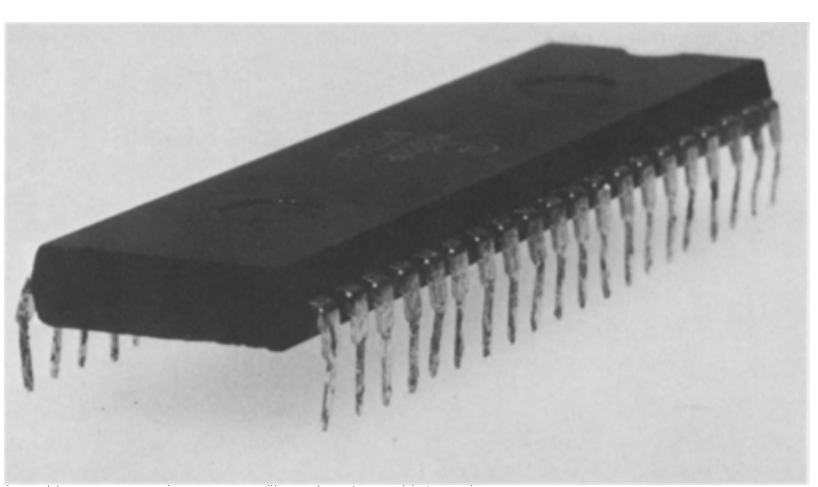

Fig. 3. Microprocessor chip, 40-pin dual in-line package

dard microcomputer systems can be used, thus reducing the development costs of electronic circuitry. Programming takes the place of the construction of electronic circuits.

\section{The Microprocessor and Microcomputer}

A microcomputer is an assembly of a microprocessor and devices to provide timing, program memory, random-access memory and interfaces for input and output operations (Fig. 4). The task of the microprocessor is to perform arithmetic or logical operations on the data it takes in and to send the results of these operations to printers, displays or to instrument interfaces that evoke control functions. The data has a digital form, that is it consists of strings of bits. A bit stands for binary digit, a digit that can only take the values one or zero. The number of binary digits that is treated as an entity is called the word length. Early microprocessors had a four-bit word length, but nowadays 8 bits and 16 bits are more common especially in applications where some calculations have to be performed. In general the efficiency of complex operations is highest in the larger word length machines as more bits are handled simultaneously.

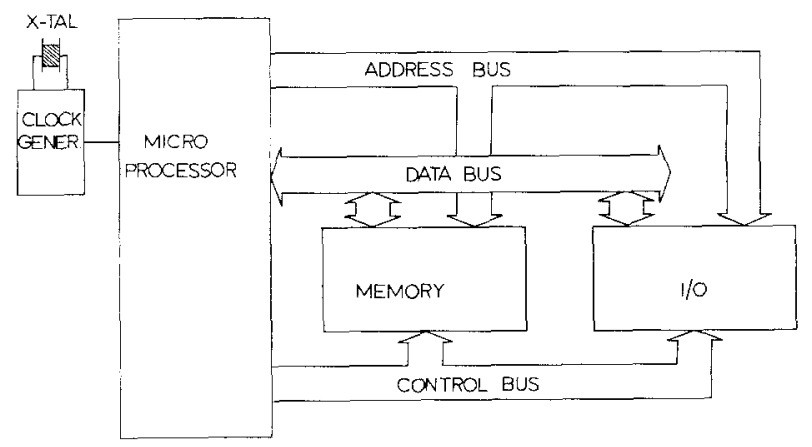

Fig. 4. Microcomputer system block diagram
Supporting the microprocessor are a number of chips of which one, the clock generator, provides the timing signals by means of a crystal-controlled oscillator. All operations somehow relate to the clock periods, so the higher the frequency of this oscillator the faster the system operates. It is noteworthy that for some microprocessor types selected species can be obtained that accept clock rates two to four times higher than the standard one. The other devices needed to constitute a complete microcomputer are memory and input/output interfaces. Microcomputer memory comes in various kinds. Read Only Memory (ROM) is meant for program storage. Its content cannot be changed, is nonvolatile, i.e., no power is needed to preserve it, and it requires a rather high initial investment for its production. So its primary use will be in highvolume productions. Programmable Read Only Memory (PROM) is also meant for program storage. It has the same properties as ROM; the only difference being its programmability with relatively simple equipment. Once programmed its content cannot be altered either. Erasable Programmable Read Only Memory (EPROM) can be used to advantage in developing new applications. It can also be programmed with relatively simple equipment, its content is nonvolatile but can be erased by exposure for about $15 \mathrm{~min}$ to a UV light source, so with some effort changes to the program can be made.

For storing changing data read/write memory (RAM) is needed. Here there are two different types: static and dynamic RAM. The static RAM will hold its data as long as power is applied to it, but dynamic RAM requires refresh signals in addition to supply voltages. The power requirements of dynamic RAM is generally lower than for static RAM but the circuits to use it are more complex because of the required refresh signals.

The $\mathrm{I} / \mathrm{O}$ interfaces constitute the communication links of the microcomputer system with the outer world. A distinction can be made between parallel and serial interfaces. Parallel interfaces allow input or output operations of a number of bits (generally equal to the word length of the microprocessor) simultaneously. This enables high transfer rates but requires a lot of connecting wires between the microcomputer system and the outer world. Slower but requiring much less cabling is serial $\mathrm{I} / \mathrm{O}$. Here the information bits are sent out or received one by one. As microprocessors operate on parallel data, parallel to serial conversion is required for output and serial to parallel conversion is required for input. Moreover protocols are needed so that the receivers can assemble the incoming bits to meaningful bit patterns. It will be clear that a transmitter and a receiver of serial data must operate at the same speed. This speed is ex- 


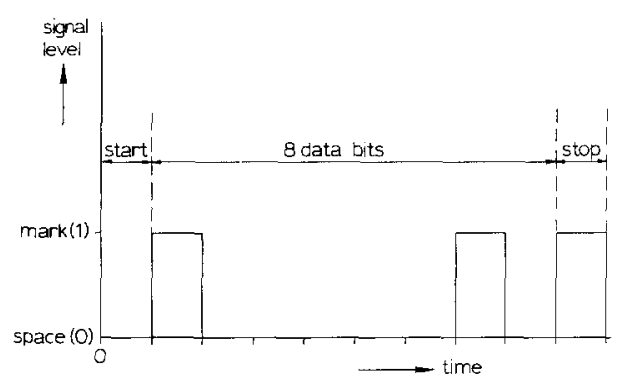

Fig. 5. ASCII character A for serial transmission

pressed in baud (bits/second). But there should also be some way to detect the start of each group of bits forming a word. In asynchronous systems the line connecting the interface to the remote instrument is normally at logical one level (mark) and the start of a word is signalled by a start bit at logical zero level (space). Thereafter follow the bits belonging to the data word, finally followed by one or two stop bits at logical one level (mark) to signal the end of the transmitted word. Figure 5 shows this type of serial transfer of data. The overhead of start and stop bits is absent in synchronous serial data transfer. Now special bit patterns (sync characters) are sent during periods that the line is idle to obtain the synchronization between transmitter and receiver. Chips specially designed for all these serial communication tasks are called USART's (Universal Synchronous/ Asynchronous Receiver/Transmitter).

The various supporting chips are connected to the microprocessor via so-called buses, sets of parallel wires along which signals are transferred. The data bus is a bi-directional path on which data is transferred from the microprocessor to memory and $\mathrm{I} / \mathrm{O}$ and from $\mathrm{I} / \mathrm{O}$ and memory to the microprocessor. The address bus is unidirectional and its state determines which memory location or $\mathrm{I} / \mathrm{O}$ interface is to be operated upon. The number of address-lines determines the maximum amount of memory locations that can be addressed. A number of 16 address lines is fairly common and can address $2^{16}=65536$ different locations.

The control bus is also unidirectional and indicates the current type of activity that the microprocessor is engaged in, i.e., memory read, memory write, $\mathrm{I} / \mathrm{O}$ read, I/O write, etc.

The basic system operation can be described as follows [1]:

1) The microprocessor issues an activity command on the control bus.

2) The microprocessor issues a binary code on the address bus to identify which particular memory location or $\mathrm{I} / \mathrm{O}$ device will be involved in the current process activity.

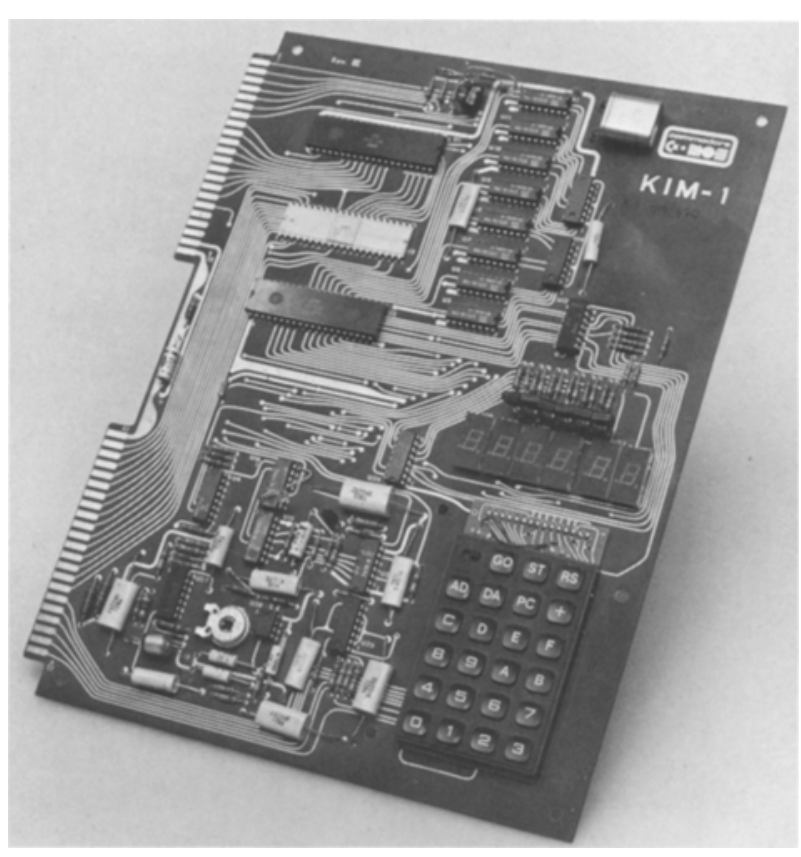

Fig. 6. Single-board computer

3) The microprocessor receives or transmits data from or to the selected memory location or $\mathrm{I} / \mathrm{O}$ device.

4) The process is repeated from step 1 with the next activity command.

The strength of this concept lies in the fact that this scheme is used to fetch commands to be executed by the microprocessor as well as to manipulate data. The total number of different commands that a microprocessor can execute is called its instruction set. Each instruction is coded as a particular sequence of 0 's and 1 's, the length of which equals the word length of the microprocessor. During operation the microprocessor fetches these instructions from memory, decodes them and takes the appropriate action.

The microcomputer configuration described so far is the minimum required, i.e., for use as an intelligent controller built into a measuring instrument. The microcomputers available in this form and for these purposes are called single-board computers (Fig. 6). For stand-alone applications packages with their own power supply in one enclosure together with some I/O interfacing to a video display and keyboard are available as personal or homecomputers.

More sophisticated are the development systems. Meant for developing microcomputer applications for mass production these development systems incorporate all the tools needed for the design and testing of programs and the simulation of small microcomputer systems. 


\section{Microcomputer Programming}

As stated above a microcomputer program consists of a set of instructions in binary machine code, i.e., sequences of zeros and ones. It is possible to write programs in this binary machine code, but it will be clear that this is very time-consuming and very likely errors will be made. The idea of using the computer to aid in programming has been a very successful one. At the lowest level of programming support are computer programs that convert mnemonics for the machine instructions and operands to binary machine code. These assemblers currently still handle the bulk of microcomputer programming. With the use of this assembly language the programming of mathematical functions still is a formidable task. Much programming effort can be saved by the use of higher-level languages like BASIC, FORTRAN, COBOL and PASCAL. One statement in these languages corresponds to a great number of machine instructions. To convert programs written in these higher-level languages to binary machine code computer programs known as compilers are used. The language PASCAL [2] is now gaining more and more terrain for microcomputer applications. It has the advantage that it allows a modular approach in the construction of programs.

A newly written program seldomly is correct the very first time. Typing errors and errors in the logic can easily be corrected with the aid of so-called editor programs.

It is not strictly necessary that program development takes place on the same type as the target microcomputer. Programs are available running on larger systems like minicomputers and main frames that produce the binary code for various types of microcomputers. These cross-compilers and cross-assemblers use the extensive facilities of the larger systems like lineprinters, large libraries of standard routines, disk storage, etc., and thus shorten the turnaround times in development.

\section{Peripherals}

Microcomputers communicate with their environment with binary-coded signals. Most of the world exterior to the microcomputers, however, is not oriented towards this type of communication. Therefore peripherals have been developed that convert various types of signals to binary information and vice versa.

The human user can exchange information with the microcomputer via teleprinters, lineprinters, typewriter keyboards and videodisplays. Currently audio sig- nals have also come into use in the form of spoken English as output and voice terminals for input.

For instrument control usually some analog signals are required. Digital to analog converters (DAC) can produce these signals from binary computer output signals. Steppermotors, however, can be controlled directly by binary signals: one pulse applied causes a specific angular movement. In most of the measurements of physical quantities transducers are employed that present the result of the measurement as an electrical voltage. These voltage values have to be converted to binary code before they can be used in a microprocessor: analog to digital converters (ADC) perform this task. Important features of these devices are speed and accuracy. Signals should be sampled at a rate at least twice their highest frequency component.

As explained before microcomputers can address only a limited amount of memory. Many applications require storage of large amounts of data, whereas in development systems a great number of standard routines should be readily available. Mass-storage devices like hard- and floppy disks handle these types of storage. Performance of these devices is determined by access time and maximum storage capacity.

\section{Microcomputer Applications in Laboratory Automa- tion}

Laboratories are concerned with the acquisition and interpretation of data. The data acquisition is carried out with the aid of measuring equipment. Automation of this equipment not only relieves the experimenter from the tedious task of taking readings from instruments, but also improves the accuracy and enables very high data rates and complicated measurement protocols. If the measured data is used to adjust experimental parameters (closed-loop control) the measurements can be optimized to speed, accuracy, etc. Apart from reducing the need for human effort and presenting new possibilities in data acquisition computerization has also a lot to offer in the field of data interpretation. Complex mathematical operations can be performed whereas presentation of the data in various report formats and in graphical form facilitates the use of the results.

A number of examples of microcomputer-controlled commercial instruments for atomic absorption, infrared and ultraviolet/visible light spectrophotometry and chromatography is given in [3]. The built-in microcomputers provide facilities that lighten the task of the operator, i.e., recallable settings of all instrument parameters, auto-adjustment of various parameters, diagnosis of malfunctioning, etc. They also sup- 


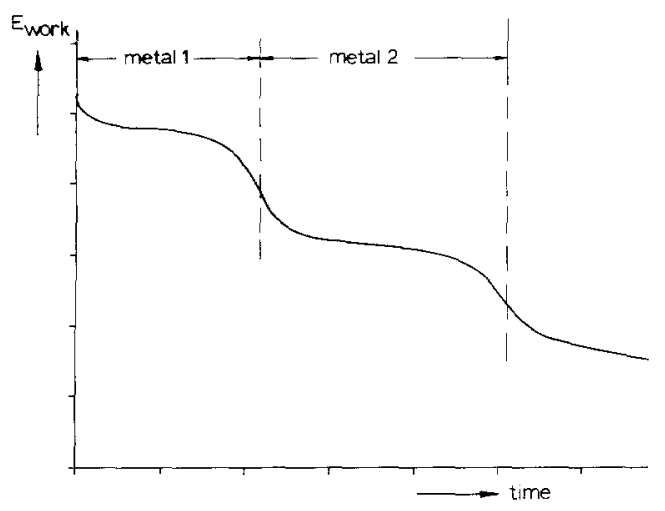

Fig. 7. Potential/time curve for potential stripping analysis

port the evaluation of the data by baseline corrections, linearization of calibration plots and peak integration.

The application of a single-board computer for the technique potential stripping analysis given by Anfält and Strandberg [4] shows that it is possible to construct elaborate equipment with standard components. To demonstrate the typical approach for the use of a microcomputer in an analytical technique this example is presented in some detail.

Potentiometric stripping analysis is a technique used in the trace analysis of metals. In this technique the sample solution of the metal ions to be determined is subjected to electrolysis. The working electrode is made of glassy carbon, a chemically rather inert material that can be polished to a high degree of smoothness. Mercury ions are added to the sample solution and thus mercury is deposited on the electrode together with the metals from the sample. This results in a thin film of mercury on the electrode in which the metals to be determined are amalgamated. After the electrolysis period the voltage source for this electrolysis is disconnected from the working electrode and the reduced metals in the mercury film are reoxidized by an oxidant into the solution. This reoxidation process changes the potential of the electrode versus a reference electrode. The resulting potential versus time curve is given in Fig. 7. The end of the reoxidation process for a given metal can be recognized by a break in this curve. The time for each metal to be stripped from the electrode is proportional to the initial concentration in the sample and electrolysis time. Figure 8 shows a schematic diagram of the complete equipment. A D/A converter is used to control the cell voltage during electrolysis. A relay serves to disconnect the D/A converter from the cell during the reoxidation, whereas an $\mathrm{A} / \mathrm{D}$ converter measures the electrode potential during this phase of the experiment. The computer program not only controls the experiment completely but also evaluates the results.

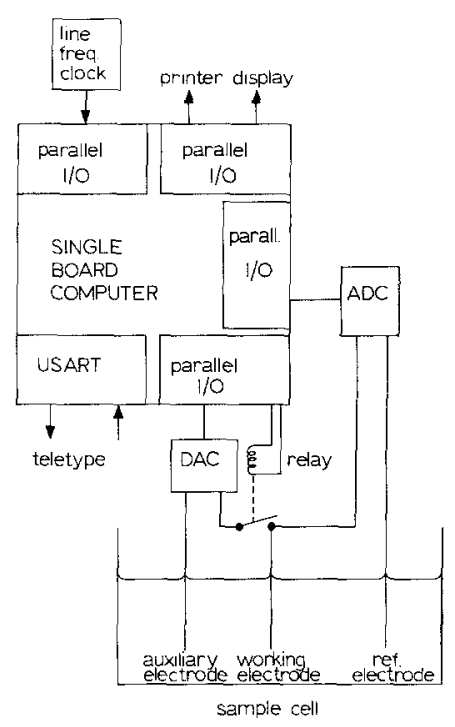

Fig. 8. Block diagram of equipment for potential stripping analysis

It is noteworthy that the authors use the higher-level programming language BASIC to ensure flexibility in changing the analysis scheme. To access the peripheral hardware special functions written in machine language were added to the standard BASIC. This technique combines the ease of programming in higher-level languages with the power of control over the hardware that machine language programming permits.

\section{Trends in the Use of Microcomputers for Laboratory Automation}

Over the years the prices of microprocessors and memory have gone down considerably and they still continue to do so. This will make computer power available in rather simple instruments. The same tendency can be observed for mass-storage devices. Magnetic bubble memories [5] are now being introduced in this field. Compared to the electromechanical devices they are more reliable and require less maintenance.

The microprocessors become more powerful, the new generation having a 16-bit word length, improved speed and elaborate instruction sets. Machine instructions for division and multiplication as well as for string handling are standard features now. More complex data processing like fast Fourier transform [6] and multiparameter curve-fitting [7] can become builtin functions of laboratory equipment with this type of microprocessors.

With the acceptance of the IEEE-488 laboratory bus standard more and more manufacturers equip their instruments with interfaces for it and the required 
software now is available for most types of microprocessors.

In contrast to research laboratories diagnostic laboratories, e.g., laboratories for process control and for clinical chemistry, have a workload of routine measurements accompanied by a heavy demand on reporting. Developments in this area include computer networks. The microcomputers dedicated to measuring instruments are coupled via minicomputers to mainframes. Experiments are completely controlled by the various microcomputers but the results are coordinated by minicomputers. Large data bases can be accessed via the links to the mainframe computers.

These developments shift the main cost of laboratory automation from the hardware to the software. Effi- ciency of the programming effort thus is of major importance. Here computer networks can also help as the vast resources of mainframe computers can be made available to the lowest level [8].

1. Intel 8080 Microcomputer Systems User's Manual, p. 3-1. Intel Corporation 1976

2. Jensen, K., Wirth, N.: Pascal User Manual and Report. BerlinHeidelberg-New York: Springer 1975

3. Franke, G.: Chemie-Technik 7, 83 (1978)

4. Anfält, T., Strandberg, M.: Anal. Chim. Acta 103, 379 (1978)

5. Hodges, D.A.: Sci. Am. 237, 130 (1977)

6. Cooley, J.W., Tukey, J.W. Math. Comp. 19, 297 (1965)

7. Wentworth, W.E.: J. Chem. Educ. 42, 96 (1965)

8. Dessy, R.E.: Anal. Chim. Acta 103, 459 (1978)

Received July 7, 1980 